\title{
THE ENDS OF MANIFOLDS WITH BOUNDED GEOMETRY, LINEAR GROWTH AND FINITE FILLING AREA
}

\author{
LOUIS FUNAR AND RENATA GRIMALDI
}

\begin{abstract}
We prove that simply connected open manifolds of bounded geometry, linear growth and sub-linear filling growth (e.g. finite filling area) are simply connected at infinity.

$M S C$ : $53 \mathrm{C} 23,57 \mathrm{~N} 15$.

Keywords: Bounded geometry, linear growth, filling area growth, simple connectivity at infinity.
\end{abstract}

\section{INTRODUCTION}

A ubiquitous theme in Riemannian geometry is the relationship between the geometry (e.g. curvature, injectivity radius) and the topology. In studying noncompact manifolds constraints come from the asymptotic behaviour of geometric invariants (e.g. curvature decay, volume growth) as functions on the distance from a base point. The expected result is the manifold tameness out of geometric constraints. This is illustrated by the classical theorem of Gromov which asserts that a complete hyperbolic manifold of finite volume and dimension at least 4 is the interior of a compact manifold with boundary. Our main result below yields tameness in the case when the filling area is finite, for those manifolds having bounded geometry and linear growth. We recall that:

Definition 1.1. A non-compact Riemannian manifold has bounded geometry if the injectivity radius $i$ is bounded from below and the absolute value of the curvature $K$ is bounded from above.

Remark 1.1. One can rescale the metric in order that $i \geq 1$ and $|K| \leq 1$ hold.

Definition 1.2. The filling area function $F_{X}(l)$ of the simply connected manifold $X$ is the smallest number with the property that any loop of length $l$ bounds a disk of area $F_{X}(l)$.

It is customary to introduce the following equivalence relation:

Definition 1.3. Two positive real functions are equivalent, and one writes $f \sim g$, if

$$
c_{1} f\left(c_{2} x\right)+c_{3} \leq g(x) \leq C_{1} f\left(C_{2} x\right)+C_{3},
$$

for positive $C_{i}, c_{i}$. By abuse of language we will call filling area the equivalence class of the filling area function.

Partially supported by GNSAGA and MIUR of Italy.

Preprint available at http://www-fourier.ujf-grenoble.fr/ ${ }^{\sim}$ funar. 
Remark 1.2. The equivalence class of the filling area function $F_{\tilde{X}}$ of the universal covering space of a compact manifold $X$ is independent of the metric we chose on $X$. In fact the filling area is a quasi-isometry invariant, and hence an invariant of the fundamental group of $X$, which we keep calling the filling area of the group.

There are two interesting classes of open Riemannian manifolds of bounded geometry:

- the universal coverings of compact manifolds. Up to quasi-isometry these manifolds are determined by their deck transformations groups. This is part of Gromov's program of classifying discrete groups up to quasi-isometry.

- manifolds of bounded geometry with linear volume growth. It is known that for any $\varepsilon>0$ there exists a metric on a given open manifold which has bounded geometry and growth less that $1+\varepsilon$. Thus super-linear growth seems to be topologically unobstructed. However there exists (see [7] 8]) a complete topological characterization of those manifolds supporting a metric of linear growth and bounded geometry, following Cheeger and Gromov ([4): these are the manifolds of finite topology at infinity. Specifically, this means that there exists a proper Morse function $\lambda$, such that the level hypersurfaces $\lambda^{-1}(n)$ are pairwise diffeomorphic for all $n \in \mathbf{Z}$.

The filling area has been studied for universal coverings and shown to be equivalent to the Dehn function of the group, measuring the complexity of the group. This topic received recently a lot of consideration (see [11 for a survey).

Remark 1.3. It is worth mentioning that sub-quadratic filling area implies linear filling area and this is equivalent to the group being word-hyperbolic. On the other hand the exponents of the polynomial filling areas fill in a dense subset in $[2, \infty)$.

This motivates the study of the filling function in the second case, as well. The definition of the filling area can be quite unappropiate for general non-compact Riemannian manifolds. Even for manifolds of bounded geometry, it might take only infinite values, in which case it does not give any valuable information about the topology. We introduce for this reason the following refined version:

Definition 1.4. Let $X$ be non-compact. The filling area function $F_{X}(l, r)$ is the smallest area of the disk in $X$ filling a loop of length $l$ lying in the metric ball $B_{X}(r)$ of radius $r$ on $X$.

Definition 1.5. The positive functions $f(l, r)$ and $g(l, r)$ are equivalent, and we write $f \sim g$, if

$$
c_{1}(l) f\left(c_{2}(l), r\right)+c_{3}(l) \leq g(l, r) \leq C_{1}(l) f\left(C_{2}(l), r\right)+C_{3}(l),
$$

for positive increasing functions $C_{i}(l), c_{i}(l)$. By filling (area) growth one means the equivalence class of the filling area function.

Remark 1.4. The filling growth is a quasi-isometry invariant.

Definition 1.6. The filling growth is said to be sub-linear if:

$$
\lim _{r \rightarrow \infty} \frac{F_{X}(l, r)}{r}=0,
$$

holds for every $l$.

Remark 1.5. If the filling area is finite then it is automatically sub-linear. 
Definition 1.7. A non-compact polyhedron $X$ is simply connected at infinity (s.c.i.), and we write also $\pi_{1}^{\infty}(X)=0$, if given a compact set $K \subset X$ there exists another compact set $L$ with $K \subset L \subset X$, such that any loop in $X-L$ is null-homotopic in $X-K$. Alternatively the map induced by the inclusion $\pi_{1}(X-L) \rightarrow \pi_{1}(X-K)$ is trivial (i.e. the zero map).

Remark 1.6. Some authors call this $\pi_{1}$-triviality at infinity or 1-LC at infinity and reserve the term s.c.i. for the special case in which $L$ can be chosen so that, in addition, $X-L$ is connected. These notions are equivalent for one ended spaces, such as contractible spaces.

Remark 1.7. The simple connectivity at infinity is an important tameness condition on the ends of the space. It has been used to characterize Euclidean space among contractible open topological $n$-manifolds by Siebenmann ([13]) for $n \geq 5$, Freedman ([] ) for $n=4$ and by Edwards ([5]) and Wall ([15]) for $n=3$ (after assuming the irreducibility to avoid the Poincaré conjecture). An earlier related result is the Stallings-Zeeman engulfing theorem (see 14), one of whose consequences is the fact that an open contractible PL $n$-manifold $(n \geq 5)$ which is s.c.i. (notice that Stallings used a slightly stronger notion of s.c.i. than that commonly used now) is PL-homeomorphic to the Euclidean space. It is thus of some interest of finding criteria which imply that a space is s.c.i.

The main result of our paper is the following topological characterization:

Theorem 1.1. A simply connected open Riemannian manifold of bounded geometry, linear growth and sub-linear filling growth is simply connected at infinity.

Corollary 1.1. An open contractible Riemannian manifold of bounded geometry, linear growth and sub-linear filling growth is diffeomorphic (only homeomorphic in dimension 4) to the Euclidean space (one needs to assume the irreducibility in dimension 3$)$.

Remark 1.8. The s.c.i. for universal coverings is a group theoretical property, which is not yet fully understood. A. Casson, V. Poenaru and M. Mihalik, among others, gave geometric conditions on the group insuring the s.c.i. Nevertheless these are not general enough in order to include all infinite 3 -manifold groups, as claimed by the uniformization conjecture.

Acknowledgements. The authors are indebted to Pierre Pansu and the referee for helpful comments and advice.

\section{A GeOMETRIC FINITENESS RESUlt}

Before we proceed let us fix the notations. We denote by $K_{M}, i_{M}$ the sectional curvature and the injectivity radius of the Riemannian manifold $M$. When $W \subset M$ is a submanifold, $i_{W \subset M}$ stands for the normal injectivity radius (i.e. the maximal radius of a tube around $W$ which is embedded in $M$ ). By $\mathrm{II}_{W}$ one denotes the second fundamental form of $W$. We fix once for all a base point $p$ on $M$, so that all metric balls in the sequel are centered at $p$, unless the opposite is explicitly stated. The Riemannian metric on $M$ induces a distance $d_{M}$ (or $d$ if no confusion arises), a volume form $\mathrm{vol}$ and a 2-dimensional area. The length of the curve $\gamma$ is usually denoted by $l(\gamma)$.

The result of this section adds some geometric control to the finiteness theorem of [7]. Specifically, we will prove below that: 
Proposition 2.1. Let $M$ be an open simply connected Riemannian manifold of bounded geometry and linear growth. There exist then:

(1) an exhaustion of $M$ by compact submanifolds $M_{j} \subset M_{j+1} \subset \ldots \subset M$,

(2) tubular neighborhoods $Z_{j}$ of $\partial M_{j}$ in $M$,

(3) constants $c, A$ and $\delta>0$ depending only on $M$,

(4) a closed Riemannian manifold $V$ of dimension one less than $M$,

such that the Lipschitz distance between $Z_{j}$ and $V \times[0, c \delta]$ is bounded by $c$, and also $d\left(p, \partial M_{j}\right) \leq A j$, for all $j$.

Proof. We need first a slight improvement of a lemma due to Cheeger and Gromov:

Lemma 2.2. Let $M$ be a Riemannian manifold of bounded geometry, $X \subset M a$ subset, $\varepsilon>0$. We denote by $T_{\varepsilon}(X)=\{x ; d(x, X)<\varepsilon\}$ the set of points having distance less than $\varepsilon$ from $X$. There exists a hypersurface $W \subset T_{\varepsilon}(X)-X$ such that:

(1) $\operatorname{vol}(W) \leq \alpha(\varepsilon)\left(\operatorname{vol}\left(T_{\varepsilon}(X)-X\right)\right)$.

(2) $\left\|\mathrm{II}_{W}\right\| \leq \beta(\varepsilon)$.

(3) $i_{W} \geq c(\varepsilon)>0$.

(4) $i_{W \subset M} \geq c(\varepsilon)>0$.

where $\alpha, \beta, c$ denote constants depending only on $\varepsilon$.

Proof. The first two requirements are granted by the approximation theorems from ([3], p.127-135) and (4]). The third part was proved in lemma 3 from [7. It remains therefore to prove that the hypersurface $W$ provided there can be supposed to satisfy also the fourth condition.

Moreover, the normal injectivity radius can be controlled by means of the second fundamental form and the curvature:

Lemma 2.3. If $\left\|\mathrm{II}_{W}\right\| \leq A$ and $K_{M} \leq A$, then $i_{W \subset M} \geq c(A)>0$ holds true.

Proof. This result seems to be well-known. One has to bound from below the distance to the closest focal point. The Jacobi theorems hold for the index form associated to the compact submanifold $W$ (see e.g. 1, 2]): thus the normal unit speed geodesic $\gamma$ has no conjugate points in some interval iff the index form is positive. The proof of the Morse-Schoenberg (or Rauch comparison) theorem extends (see 1], p.79) without essential modifications to this setting (see also [12, p.172175). Otherwise one can use the more general Rauch comparison theorem sketched in (1], Remark 3.4., p.135). Effective estimates for the constant are given in 10 where only positively curved manifolds are considered, but the arguments work for curvature bounded from above.

This yields the claim of lemma 2.2

A result similar to lemma 2.2 has been used in 7, in order to obtain the existence of an exhaustion $M_{j}$ whose boundaries $\partial M_{j}$ belong to a finite family of diffeomorphism types. One has then to see that the Lipschitz distances are uniformly bounded and further that the distance to the boundaries grows linearly.

The lemma 2.2 yields uniform lower bounds (away from zero) for the normal injectivity radii $i_{\partial M_{j} \subset M}$, and the fact that the metrics induced on the manifolds $\partial M_{j}$ (which are pairwise diffeomorphic) are at uniformly bounded Lipschitz distance one from each other. This implies the first assertion from proposition 2.1. 
In what concerns the second claim of proposition 2.1 we follow closely the proof of the main result from [7. Specifically we can state:

Lemma 2.4. There exists an exhaustion $\left\{M_{j}\right\}$ and constants $c_{i}=c_{i}(M)$ such that:

(1) the boundary manifolds $\partial M_{j}$ inherit metrics fulfilling

$$
\left|K_{\partial M_{j}}\right| \leq c_{1}, i_{\partial M_{j}} \geq c_{2}>0, \operatorname{vol}\left(\partial M_{j}\right) \leq c_{3}, \text { for all } j,
$$

(2) the boundary $\partial M_{j}$ is sandwiched between two metric spheres, namely

$$
c_{4} j \leq d\left(p, \partial M_{j}\right) \leq c_{5} j \text {, for all } j .
$$

Proof. In order to obtain (2) it suffices to improve the sub-lemma 2.1 from ([7], p.853), as follows:

Lemma 2.5. Let $A(n)=B_{M}(n+1)-B_{M}(n)$ be the annuli of unit width, where $B_{M}(n)$ denotes the ball of radius $n$ centered at $p$. Then there exists a sequence $n_{k}$ and constants $c, C$ such that

(1) $\operatorname{vol}\left(A\left(n_{k}\right)\right) \leq c$,

(2) $n_{k} \leq C k$.

Proof. The linear growth hypothesis is that $\operatorname{vol}\left(B_{M}(n)\right) \leq a n$, for some constant $a$. Let $S=\left\{n \in \mathbb{Z}_{+} ; \operatorname{vol}(A(n)) \leq 2 a\right\}$. Assume that $\operatorname{card}(S \cap\{1,2, \ldots, N\})<\frac{N}{2}$. Then $\operatorname{vol}\left(B_{M}(N)\right)>(2 a) \frac{N}{2}=a N$, contradiction. This shows that one can take $c=C=2$ above.

In particular there exists an exhaustion $M_{j}$ with the property that all $\partial M_{j}$ are diffeomorphic and moreover $d\left(p, \partial M_{j}\right) \leq A C j$, where $A$ is the number of diffeomorphism types of manifolds satisfying the first condition in lemma 2.4 (and $A$ being finite by Cheeger's finiteness theorem). This proves lemma 2.4

As already remarked this lemma ends the proof of proposition 2.1

\section{The PROOF OF THE THEOREM}

Assume that we fixed an exhaustion $M_{j}$ and the tubular neighborhoods $Z_{j}$ like in the proposition 2.1 One knows that $Z_{j}$ is canonically identified with a cylinder $\partial M_{j} \times\left[0, r_{j}\right]$, where all $r_{j}$ are bounded from below by some $\delta>0$.

Lemma 3.1. There exists a constant $c_{6}$ with the following property. For any 2-disk $D$ transverse to $Z_{j}$, such that $D \cap Z_{j}$ is not null-homotopic (i.e. $D \cap M_{j} \times\{t\}$ is not null-homotopic, for any $\left.t \in\left[0, r_{j}\right]\right)$ we have area $\left(D \cap Z_{j}\right) \geq c_{6}$.

Proof. Let $c l_{0}$ be the 1-systole of $V$, i.e. the length of its smallest closed geodesic. By proposition 2.1 the length of each non-trivial component of $D \cap \partial M_{j} \times\{t\}$ is bounded from below by $l_{0}$. Then the projection $p: \partial M_{j} \times[0, \delta] \rightarrow[0, \delta]$ is decreasing the length, i.e. $\|\nabla p\| \leq 1$. We take then $c_{6}=\delta l_{0}$. In fact the coarea formula states that:

$$
\begin{aligned}
& \operatorname{area}\left(D \cap \partial M_{j} \times[0, \delta]\right)=\int_{0}^{\delta} d t \int_{D \cap \partial M_{j} \times\{t\}} \frac{d s}{\|\nabla p\|} \geq \\
& \geq \int_{0}^{\delta} d t \int_{D \cap \partial M_{j} \times\{t\}} d s \geq \int_{0}^{\delta} l\left(D \cap \partial M_{j} \times\{t\}\right) d t \geq \delta l_{0}
\end{aligned}
$$


Proof of the theorem. Suppose that the contrary holds so that $M$ is not simply connected at infinity.

Lemma 3.2. There exists then a compact $K$ such that the maps induced by the inclusions $\iota_{j}: \pi_{1}\left(\partial M_{j}\right) \rightarrow \pi_{1}(M-K)$ are non-zero, for all large enough $j$.

Proof. There exists a compact $K$ such that arbitrary far loops bound only disks touching $K$. If $K \subset M_{j_{0}}$ consider loops $l_{j}$ outside $M_{j}$ with this property. Any disk $D_{j}$ bounding $l_{j}$ should intersect $K$ hence $\partial M_{j}$. One puts the disk $D_{j}$ in general position with respect to $\partial M_{j}$. It follows that at least one loop component of $\partial M_{j} \cap D_{j}$ is not null-homotopic. Further the image of at least one loop component in $\pi_{1}(M-K)$ is non-zero. In fact, otherwise $l_{j}$ would bound a disk not touching $K$, when capping off the null-homotopy disks of these loops with the annuli $D_{j} \cap$ $M-M_{j}$. This contradicts the choice of $l_{j}$.

Recall that all $\partial M_{j}$ are diffeomorphic to the closed manifold $V$. If $a_{1}, \ldots, a_{N}$ is a system of generators of $\pi_{1}(V)$, one has then an induced system of generators for $\pi_{1}\left(\partial M_{j}\right)$, which we keep denoting by the same letters. There exists therefore $k \in\{1,2, \ldots, N\}$, such that the images $\iota_{j}\left(a_{k}\right)$ are non-zero, for all large enough $j$. Let now $a_{k, j}$ denote a loop in $\partial M_{j}$ representing $a_{k}$ in $\pi_{1}\left(\partial M_{j}\right)$.

Lemma 3.3. There exists some $c_{7}$ such that representatives loops $a_{k, j}$ can be chosen to be of length uniformly bounded by $c_{7}$, for all $k$ and $j$.

Proof. This is a consequence of the fact that $\partial M_{j}$ are at uniformly bounded Lipschitz distance from $V$.

Consider now a disk $D_{j}$ in $M$, filling the loop $a_{k, j}$.

Lemma 3.4. For large enough $j$ we have area $\left(D_{j}\right) \geq \frac{c_{6}}{2} j$.

Proof. One can assume that $D_{j}$ is transverse to all $Z_{i}$ 's. By hypothesis $D_{j} \cap K \neq \emptyset$. Let $j_{0}$ be the minimal number with the property that $K \subset M_{j_{0}-1}$. We claim that for all $i \in\left\{j_{0}, \ldots, j\right\}$ the intersections $Z_{i} \cap D_{j}$ are not null-homotopic. Otherwise we could replace the disk $D_{j}$ by a more economical one, contained in $M_{j}-M_{j_{0}}$, which would contradict our choice of $j_{0}$. Further, by using lemma 3.1 one derives:

$$
\operatorname{area}\left(D_{j}\right) \geq \sum_{i=j_{0}}^{j} \operatorname{area}\left(D_{j} \cap Z_{i}\right) \geq c_{6}\left(j-j_{0}\right) .
$$

This proves the lemma 3.4

Eventually we observe that the loops $a_{k, j}$ have uniformly bounded length and stay at distance $c_{5} j$ far from the base point, because $a_{k, j} \subset \partial M_{j}$. In particular the filling growth is linear in the radius. This contradicts our hypothesis, and hence the theorem follows.

\section{A counterexample to the converse}

The metrics of bounded geometry on open s.c.i. manifolds need not have a sub-linear filling area, as the following example shows:

Example 4.1. There exists a Riemannian metric $g$ of bounded geometry on $\mathbf{R}^{n}$ (for $n \geq 3$ ) such that

$$
\lim _{r \rightarrow \infty} \frac{F_{\left(\mathbf{R}^{n}, g\right)}(l, r)}{r} \neq 0 .
$$


Proof. The method consists in modifying a decomposition of $\mathbf{R}^{n}$ into compression bodies by adding trivial cylinders of sufficiently large length. A loop bounding in a compression body can be translated along the cylinder and thus the bounding disk for the new loop is the union of the former with a long cylinder. In particular we can achieve a linear area function for this type of loops.

Specifically let us focus on $n=3$, and set $T_{j}$ for an increasing family of solid tori, such that:

(1) $T_{j+1}-\operatorname{int}\left(T_{j}\right)$ is a product $T^{2} \times[0,1]$ for all $j$ but an infinite sequence $A=\left\{j_{1}, j_{2}, \ldots, j_{k}, \ldots\right\} \subset \mathbb{Z}_{+}$. Here $T^{2}$ stands for the 2 -torus.

(2) if $j \in A$ then the inclusion $T_{j} \hookrightarrow T_{j+1}$ is trivial up to isotopy, and thus $T_{j}$ is contained in a ball $B^{3}$ embeded in $T_{j+1}$. Hence $T_{j_{i}+1}-\operatorname{int}\left(T_{j_{i}}\right)=V^{3}$ has a fixed diffeomorphism type, and it is not diffeomeorphic to $T^{2} \times[0,1]$. In particular the map $\pi_{1}\left(\partial T_{j_{i}+1}\right) \rightarrow \pi_{1}\left(T_{j_{i}+1}-\operatorname{int}\left(T_{j_{i}}\right)\right)$ is not zero.

We consider a metric structure $g$ on each $T_{j+1}-\operatorname{int}\left(T_{j}\right)$, which is a product along the boundary (corresponding to each one of the two models, $V^{3}$ or $T^{2} \times[0,1]$ ), with isometric boundaries at unit distance from each other. We obtain a metric on $\mathbf{R}^{3}-T_{1}$, which can be completed by capping off with a Riemannian structure on $T_{1}$. This metric has linear growth.

Let $c$ be a loop on $\partial T_{j_{i}}$, which is not null-homotopic and does not bound in $\mathbf{R}^{3}-T_{j_{i}}$, and thus it is not a longitude. Then any disk $D^{2}$ filling $c$ has a component in $T_{j_{i}}$, and the innermost circle component of $D^{2} \cap \partial T_{j_{i}}$ is still homotopically non-trivial. We can suppose that this component is actually $c$, and hence that $c$ bounds in $T_{j_{i}}$. It follows that $D^{2} \not \subset T_{j_{i}}-T_{j_{i-1}+1}$, because $T_{j_{i}}-T_{j_{i}+1}$ is a cylinder $T^{2} \times\left[0, j_{i}-j_{i-1}\right]$ retracting on $\partial T_{j_{i}}$. Therefore $D^{2}$ intersects $T_{j_{i-1}}$ and thus $D^{2}$ intersects non-trivially (i.e. along loops which are not null-homotopic) the $j_{i}-j_{i-1}$ intermediary tori $\partial T_{j}$ with $j_{i-1}+1 \leq j \leq j_{i}$. Since these tori have uniformly bounded normal injectivity radii (thus neighborhoods isometric with $T^{2} \times[0, \delta]$ ) we obtain that $\operatorname{area}\left(D^{2}\right) \geq \delta\left(j_{i}-j_{i-1}\right)$. Now $\partial T_{j}$ is at distance $j-1$ from $T_{1}$ and thus we find that:

$$
\lim _{r \rightarrow \infty} \frac{F_{\left(\mathbf{R}^{3}, g\right)}(l, r)}{\delta r} \geq \lim _{i \rightarrow \infty} \frac{j_{i}-j_{i-1}}{j_{i}} .
$$

By choosing $j_{i}$ growing fast enough we can insure that the right hand limit is 1 .

\section{Comments}

There exists a further refinement of the filling area function, as follows. Set $f_{X}(l, r ; \lambda)$ for the smallest area of the disk in $X$ filling an arbitrary loop of length $l$ lying in the annulus $B_{X}(r)-B_{X}(\lambda r)$ of $X$. The function $f_{X}(l, r ; \lambda)$ need not being increasing anymore. The filling growth is said to be weakly sub-linear if

$$
\lim _{r \rightarrow \infty} \inf \frac{f_{X}(l, r ; \lambda)}{r}=0, \text { for any } 0<\lambda \leq 1 .
$$

The growth of $f_{X}(l, r ; \lambda)$ is not a quasi-isometry invariant since the modulus of the annulus might be changed by a quasi-isometry. However the property of having a weak sub-linear filling area is a quasi-isometry invariant, as it can be easily checked. It is not difficult to see that the proof of our theorem actually shows that a manifold of bounded geometry and linear volume growth whose filling growth is weakly sublinear should be simple connected at infinity. 


\section{REFERENCES}

[1] I. Chavel, Riemannian geometry - a modern introduction, Cambridge Tracts in Math., 108, Cambridge University Press, 1993.

[2] J. Cheeger and D. Ebin, Comparison theorems in Riemannian geometry, North Holland, 1975.

[3] J. Cheeger and M. Gromov, On the characteristic numbers of complete manifolds of bounded curvature and finite volume, Differential geometry and complex analysis, 115154, H.E.Rauch Memorial volume, (I. Chavel and H. M. Farkas Ed.), Springer, Berlin, 1985.

[4] J. Cheeger and M. Gromov, Chopping Riemannian manifolds, Differential geometry, A Symposium in Honor of Manfredo do Carmo, (H. Blaine Lawson, Jr. and Keti Tenenblat, Editors), 85-94, Pitman Monogr. Surveys Pure Appl.Math., 52, Longman Sci. Tech., Harlow, 1991.

[5] C.H. Edwards, Open 3-manifolds which are simply connected at infinity, Proc. A.M.S., 14(1963), 391-395.

[6] M. Freedman, The topology of four-dimensional manifolds, J. Diff. Geometry, 17(1982), 357-453.

[7] L. Funar and R. Grimaldi, La topologie à l'infini des variétés à géométrie bornée et croissance linéaire, J.Math.Pures Appl., 76(1997), 851-858.

[8] R. Grimaldi, Croissance linéaire et géométrie bornée, Geom. Dedicata 79(2000), no. 3, 229-238.

[9] M. Gromov, Volume and bounded cohomology, Publ.I.H.E.S. 56 (1982) 5-99.

[10] R. Hermann, Focal points of closed submanifolds of Riemannian spaces, Indag. Math. 25(1963), 613-628.

[11] A.Yu. Olshanskii, M.V. Sapir, Length and area functions on groups and quasiisometric Higman embeddings, Internat. J. Algebra Comput. 11 (2001), no. 2, 137-170.

[12] M.M. Postnikov, The variational theory of geodesics, W. B. Saunders Co., Philadelphia-London, 1967.

[13] L.C. Siebenmann, On detecting Euclidean space homotopically among topological manifolds, Inventiones Math., 6(1968), 263-268.

[14] J. Stallings, The piecewise-linear structure of Euclidean space, Math.Proc. Cambridge Phil. Soc., 58(1962), 481-488.

[15] C.T.C. Wall, Open 3-manifolds which are 1-connected at infinity, Quart.J.Math.Oxford, Ser. (2), 16(1965), 263-268.

Institut Fourier BP 74, UMR 5582 CNRS, Université de Grenoble I, 38402 SaintMartin-D'HÈres Cedex, France

E-mail address: funar@fourier.ujf-grenoble.fr

Dipartimento di Matematica ed Applicazioni, Facolta di Ingegneria, Università di Palermo, Viale delle Scienze, 90128, Palermo, Italia

E-mail address: grimaldi@math.unipa.it 